\title{
MALÌA DI LUIGI CAPUANA. IL GERME DELLA MODERNITÀ IN «UN CASO DI PASSIONE PATOLOGICA»
}

\author{
MALİA DI LUIGI CAPUANA. MODERNITY'S SOURCE IN «UN CASO DI \\ PASSIONE PATOLOGICA»
}

\section{RIASSUNTO:}

Malìa è un'opera teatrale verista innovativa . $\mathrm{E}^{\prime}$ una delle opere più felici di Capuana, cui protagonista è l'universo femminile con lesue passioni. L'articolo contiene riflessioni e commenti di vari autori sull'opera, tra i cuali Verga.

\section{PAROLE CHIAVE:}

Capuana, dramma, Malìa, opera, passione, verismo.

\section{ABSTRACT:}

Malia is a innovative play that belongs to Italian verismo. It is one of the happiest works of Capuana, whose protagonist is the feminine world with its passions. The article includes remarks by many other authors, among which Verga.

KEY WORD:

Capuana, drama, Malìa, play, passion, Italian "verismo". 
Vagheggiato negli anni delle «illusioni sublimi» dell'acerba giovinezza, tentato con farraginosa competenza sin dagli esordi del «noviziato di commediografo», sottoposto al vaglio dell'esame ermeneutico nel corso dell'apprendistato critico, rinnovato nelle convenzioni tecniche, sfidato nel conformismo morale, il teatro costituisce una presenza costante dell'affollata, operosa officina di Luigi Capuana. Vi si accampa, protagonista assoluto, l'universo femminile con le sue convulsioni psicologiche, con le sue accese passioni, col suo ipertrofico sentire. Insieme incipit ed explicit della sua parabola umana ed artistica, scorrendo la produzione teatrale del mineolo è possibile tracciare l'evoluzione di una poetica, delineare un percorso, talora accidentato, specchio fedele del periodo di crisi e di lento mutamento di gusto vissuto dalla letteratura italiana alla fine dell'Ottocento, dallo sfaldarsi degli ideali eroici del Risorgimento, all' affermazione del credo positivista agli albori del simbolismo e del decadentismo. Un percorso attraversato da un'ansia di sperimentazione che darà corpo ad una drammaturgia come terreno di applicazione di una teoresi sul teatro alla quale il mineolo affiderà gli esiti più maturi della sua riflessione critica, dall'elaborazione del verismo al suo superamento con l'approdo ad una sensibilità di tipo novecentesco.

Definita da Giovanni Verga un «vero gioiello» e da Federico De Roberto «un'opera forte e bella», Malia, il testo teatrale più felice di Capuana, col quale soleva esordire Giovanni Grasso, «il più grande attore tragico del mondo» (Muscarà, Zappulla, 1995) è compiuta sintesi dell'impegno interpretativo, della pratica creativa e della prova scenica di un intellettuale che proprio a partire dal teatro ha seguito un itinerario coerente in direzione del rinnovamento della cultura nazionale. Con l'approdo nella Firenze capitale d'Italia, laddove si reca col convincimento di divenire «niente meno che lo Shakespeare d'Italia», constatato l'anacronismo dei suoi drammi in versi ispirati alla causa unitaria raffrontati con l'avanguardia teatrale francese, il mineolo abbandona l'ambizioso progetto a favore di un esercizio esegetico che gli avrebbe consentito di «prepararsi convenientemente» alla carriera drammatica. Pubblicando sulle colonne de «La Nazione» recensioni di spettacoli di autori italiani e stranieri, Capuana avvia così un'analisi sul teatro del tempo che si palesa come la sede privilegiata per la formulazione dei canoni del verismo: il metodo impersonale, la necessità dello studio dal vero, il nesso arte-vita e ancora l'idea hegeliano-demeisiana delle forme artistiche come organismo vivente, la teoria della forma. Enunciati organici al suo sistema estetico che fisserà, con stringente argomentazione, nell'introduzione al volume Teatro italiano contemporaneo ove raccoglierà i saggi di critica teatrale. Un verismo che Capuana vorrebbe applicare prima di tutto al teatro in virtù di quel genere dialogato che consente una più completa eclissi dello scrittore, condividendo con Federico De Roberto l'idea secondo cui «l'impersonalità assoluta non può conseguirsi che nel puro dialogo, e l'ideale della rappresentazione obbiettiva consiste nella scena come si scrive pel teatro» (De Roberto, 1890: VI).
Ma il «vento della così detta cultura moderna» (Capuana, 1888: IX), che soffiava forte negli ambienti culturali fiorentini, vigorosamente disperde le aspirazioni drammatiche del mineolo, soprattutto adesso che il «mortifero veleno della novella e del romanzo» (Capuana, 1888: XXI), evocato dalla lettura di Balzac e Diderot, lo indirizza verso una forma più moderna, decretando la condanna del dramma storico. È così che Capuana si rivolge alla narrativa verificando la validità di quei principi, mutuati dagli scrittori francesi, che porrà alla base della sua produzione letteraria. A popolarne l'immaginario l'ampia casistica psicologica di donne avvinte da forti passioni che vede il suo esordio nella silloge di novelle Profili di donne, un volume, osserva Attilio Momigliano, che fa dello scrittore siciliano «un iniziatore di quella psicologia intricata ed ombrosa che, limitata ancora in lui e frenata da un certo abito scientifico, doveva, discendendo in parte dall'immenso Dostoevskj, dilagare e diventare sempre più malinconica e trista $\mathrm{e}$ inafferrabile in D'Annunzio e nel romanzo che va da Borgese a Moravia» (Momigliano, 1962: 556).

Quella propensione per il caso clinico, consegnato all'anamnesi dello «scienziato dimezzato», variamente modulandosi, costituirà l'autentica cifra del mineolo riproponendosi nelle prove migliori del narratore come del drammaturgo, frattanto tornato al teatro nella seconda metà degli anni ' 80 sperimentando il modello della novella dialogata con Il piccolo archivio, provandosi in un disordinato esercizio definito con la singolare metafora di «diarrea drammatica», provocando l'etica borghese con l'adattamento scenico del «bel soggetto, ma scabrosissimo» tratto dal suo più famoso romanzo accolto da un «urlo d'indignazione». Un filo rosso, quello dell'esplorazione di nevrosi femminili, che va da Giacinta a Ribrezzo a Profumo al Marchese di Roccaverdinagiungendo fino a Malìa dopo un lungo processo di revisione di quei postulati teorici che alla fine del secolo recano il segno del progressivo allontanamento dallo scientismo di marca zoliana. Lo documenta la vicenda scenica di Malìa, simbolo di un malessere psicologico che prelude all'inquietudine esistenziale di cui la letteratura del Novecento esibirà più vistosi esempi.

Il 25 novembre 1891 il mineolo comunica all'amico De Roberto di avercompletato Malia, sottolineandone le caratteristiche innovative che aboliscono quella «convenzione», tipica del dramma di stampo ottocentesco, già condannata nelle cronache fiorentine

ieri alle 2 e 40 p.m. misi la parola fine alla commedia, e domani la leggerò a Cesare Rossi. Io sono contento del mio lavoro: mi pare d'aver scritto una cosa teatralissima, drammaticissima, senza nessuna convenzione nei caratteri, nei sentimenti, nell parte tecica della scenge na parte tecnica della sceneggiatura... Resta poi a vedere se quel che pare alla mia
coscienza sia tale davvero (Zappulla Muscarà, 1984: 334).

Entusiasta la reazione di De Roberto che così gli scrive: 
Mio caro Luigi, vorrei poterti abbracciare, sarebbe l'unico modo col quale esprimerti il piacere immenso provato nel leggere la tua Malia, l'ammirazione per quest'opera forte e bella che è fra le tue migliori e che se la p... fortuna dirà di sì, darà finalmente quel successo del quale sei meritevole. Tu hai messo le mani sopra un argomento interessantissimo, pieno di carattere, di colore locale, di teatralità un argo'h on belli. Non belli. Non eslo mani, saltarti al collo, stringerti la pancia. Bravo! Bravo! Bravo! (Zappulla Muscarà, 1984:

Eil Verga, del quale Capuana, «ansiosissimo», ripetutamente aveva sollecitato il giudizio:

Ed ora veniamo alla tua Malia, coll'accento sull'i, che da quindici giorni ho sulle labbra e dinanzi agli occhi, un vero gioiello. Porco, infame, assassino! mi hai fottuto una o due scene della Lupa, ma è la più bella cosa che tu abbi fatto, la più bella cosa che si sia fatta da qualche tempo a questa parte, e sarà un vero successo. L'ho data ad Eisenschits per la traduzione tedesca, e a Giacosa da leggere; per la gran mania che ho di vedere che la trovino bella come la trovo io tutti quelli di cui ho stima Perdonami l'indiscrezione, se mai. [...] Sono contento come se l'avessi fatta io (Raya, 1984: 340).

Ambientato sullo sfondo di una Sicilia ancestrale, terra di passioni forti, di credenze popolari, edificato sull'ordinamento patriarcale della società, regolato dalle ferree leggi dell'onore, della famiglia e della 'roba', il testo capuaniano trae alimento dalla rivisitazione del microcosmo paesano, cuil lo riconduce pure il successo del «laboratorio» verghiano, attingendone materia d'arte. Assumendo a oggetto di rappresentazione un nucleo familiare improntato ai valori su cui si regge la piccola borghesia paesana dell'Ottocento, Capuana vi introduce l'elemento perturbatore della passione illecita che viene repressa poiché potenzialmente destabilizzante l'ordine precostituito e innesta, sull'intreccio della storia d'amore di Cola e Nedda, cui fa riscontro quella di Ninu e Jana, la relazione incestuosa di Cola e Jana, definendo un triangolo amoroso il cui vertice, Jana, costituisce un personaggio nuovo nell' ambito del consolidato schema dell'adulterio. Combattuta tra il rispetto del patto matrimoniale e il trasporto per il cognato, la protagonista dell'opera non tradisce con la leggerezza che connota altre figure femminili del panorama letterario del tempo e così rifiuta il promesso sposo Ninu perché 'ammaliata' da Cola, la passione per il quale, interiorizzata, provoca dei turbamenti nella sua psicologia somatizzati in un'esaltazione nervosa che, sia pure trovando giustificazione nella superstizione della fattura, rivela il male oscurod un'interiorità sempre più insondabile. Nell'espediente della malìa e nel gesto tradizionale dell'omicidio di Cola per mano di Ninu la risoluzione del conflitto scaturito dalla trasgressione delle norme condivise. E si ripete l'eco che risuona dalla Cavalleria rusticana al grido di «Hanno ammazzato compare Turiddu! Hanno ammazzato compare Turiddu!».
Ma se il tragico epilogo pone Malia nel solco tracciato dal fortunato dramma verghiano (con una filiazione diretta, larvatamente rivendicata dall' autore, anchedalla Lupa), è nel personaggio di Jana che bisogna individuare un fattore di rottura della codificazione del genere. Come ha osservato Guido Nicastro «è questo l'elementonuovo ed originale di Malia: se il contrasto fra Cola e Ninu e l'omicidio finale rientrano perfettamente nella struttura tradizionale del teatro siciliano, il dramma che si svolge, con esito fisiologico distruttivo, nell'animo di Jana tra dovere e sentimento, tra senso dell'onore e passione, costituisce un elemento di novità nella storia di quel teatro» (Nicastro, 1978: 125). Inquieta, instabile, isterica, vivendo con tormentata consapevolezza un turbamento interiore che viola i legami consacrati dalla tradizione e dalla fede, Jana è straziata artefice dell'inesorabile distruzione cui va incontro il suo nucleo familiare. Ne costituisce il veicolo la passione che, osserva Capuana, «esploda in alto o in basso, tra creature popolane o aristocratiche, è cosa elevata, concentramento di forze, complicazione di sentimenti, energia, lotta, catastrofe, dramma insomma» (Capuana, 1898: 183). Anche al critico teatrale Stanis Manca così, al riguardo, aveva precisato:

In Malia ho voluto rappresentare obbiettivamente un caso di passione patologica che forse non è assolutamente regionale, perché la superstizione della fattura è diffusa anche in altre province italiane. Siccome io non amo l'astratto o l'indeterminato, ho cercato di rendere così nel mio lavoro in qual modo si svolgerebbe un tal caso quaggiù dove le passioni sono più calde e diciamo anche più primitive (Oliva, 1999: 109)

Riconducibile al pathos della gloriosa tradizione mediterranea, il motivo della passionalità, potente detonatore della carica emozionale, efficace propulsore dell'azione drammatica, fa di Malìun'opera fortemente connaturata ai «canoni della tragedia greca» (Morea, 1995: XIII) da cui la cultura siciliana direttamente discende. Una partecipazione alle forme del tragico, cui aderisce la ricca coralità di figure di contorno, attestata da una composita "campionatura delle marche di tragicità» (Muscariello, 2005: 86) che amplifica la valenza di dramma individuale e familiare. Ma al consueto dispositivo della passione clandestina in Malia si affianca un caso di psicopatologia passionale che sposta il nucleo drammatico sull' introspezione interiore della protagonista, su quell'ossessione, in cui risiede il fascino e l'originalità del testo, che rivela la spia di una diversa visione e dell'individuo e dell'arte.

Privata del sentimentalismo di tanta pratica letteraria, la passione diviene sintomo di una patologia, non più riconducibile a deterministiche variabili di race, milieu, moment (Jana, infatti, non è il risultato dell'ambiente degradato che aveva plasmato Giacinta bensì di una comunità fondata sul rispetto di valori condivisi), che è rivelatrice di un intricato processo psichico e insieme di inquietanti zone buie della vita emotiva ed 
inconscia. Il dramma enigmatico di Jana, l'ineffabile spasmo che le fa sfiorare la follia di cui è spia il linguaggio sincopato, disarticolato, allusivo, non istituisce un acclarato caso clinico bensì un oscuro conflitto dell'anima che gli strumenti dell'indagine positivista non possono decifrare. All'analisi scientifica dell'uomo frutto di ereditarietà succede dunque l'inesplorabile profondità degli abissi della coscienza e il dottor Follini, schermito dalla macchietta del mago don Saverio Teri che ne smorza l'energia dirompente dislocandola sul piano del bozzettismo, cede il passo allo scrutatore di anime, a quello scandaglio dell'inconscio che apre la via alla scissione esistenziale. Un esito questo certamente inaudito rispetto alle premesse teoriche del «feroce naturalista» che, se lascia indovinare l'influsso dell'irrazionale, nondimeno finisce con il riparare nell'ambito di una razionalità rassicurante, quantunque contaminata da inveterati retaggi popolari, tipica della visione del mondo della borghesia paesana.

Ma l'ancoraggio all'interiorità è rivelatore pure di una diversa concezione dell'arte affacciatasi alla meditazione estetica del mineolo sul crinale del secolo. Impegnato nella faticosa ricerca di una formula stilistica aderente alla maturità di un letterato sempre più insofferente delle rigide classificazioni (tanto che a Éduard Rod, nel 1883, aveva dichiarato di tenere «una gamba nel positivismo e un'altra nell'idealismo egheliano») Capuana guarda a più vasti orizzonti interpretativi al fine di mediare naturalismo fisiologico e psicologico, verità e verismo di maniera, abbandonando ogni forma di «giacobinismo critico ed artistico» (Madrignani, 1970: 236) a favore di un ideale dell'arte vivificato da nuove tendenze. Sia pure confermando il ruolo dello scrittore contemporaneo, che «ruba il mestiere al psicologo, al fisiologo, al professore di scienze sociali. Non già che predichi, che dimostri, che voglia far la lezione; ma egli scortica vivi vivi i suoi personaggi, ma egli pianta il bisturi in quelle carni palpitanti con la stessa spietata indifferenza di un anatomico» (Capuana, 1885: XLIII), il mineolo ne ridimensiona l'autorità per sottolineare di contro le «due divine facoltà: la fantasia, l'immaginazione, che potrebbe anche darsi siano un'identica cosa» (Capuana, 1885: XLIX), ripristinando quella componente idealistica, già operante nella riflessione giovanile, che si manifesta con evidenza nei racconti di Ribrezzo e in Profumo, cui attende, non a caso, nello stesso torno di tempo della stesura di Malìa. Fra i «documenti umani» e lo «spiraculum vitae» Capuana conia così una formula composita, delatrice di una stagione di profonda trasformazione, di un'intelligenza vigile e versatile sempre pronta a cogliere i mutamenti epocali, proteso esclusivamente a ripetere, «nella forma letteraria, il segreto processo della natura», a rigettare quegli «"ismi” contemporanei» che incarnano astratte quanto imperiose tassonomie, fino ad affermare «io, caso mai, sono naturalista, verista, quanto sono idealista e simbolista: cioè tutti i concetti o tutti i soggetti mi sembrano indifferenti per l'artista ed ugualmente interessanti, se da essi egli riesce a trar fuori un'opera d'arte sincera» (Capuana, 1899: 250). Capuana, con Giuseppe Antonio Borgese, «non appartenne mai ad un sistema o ad una scuola; non fece mai sacrificio della sua personalità a una fede o ad un metodo, ma si servì, con gioconda agilità, dei metodi e delle mode per compiacere al suo prepotente istinto narrativo» (Borgese, 1928: 176). Unico obiettivo «far del vivo», un'esigenza intrinseca al suo credo estetico sin dalla formulazione del verismo («io innanzitutto amo in arte la vita. Quando l'artista riesce a darmi il personaggio vivente davvero non so chiedergli altro e lo ringrazio. Mi pare ch'egli m'abbia dato tutto quello che dovea. E al pari del personaggio amo viva l'azione») (Capuana, 1872: XI), ma che ora si carica di un significato inusitato, coniugandosi il soffio vitale a quella tensione verso l'interiorità cui lo riconduce la nuova, moderna sensibilità artistica. «Persone vive» considerava il mineolo i personaggi del sodale Verga, «perché sono nello stesso tempo esteriori e interiori; perché ogni loro parola, ogni loro atto rivela uno stato d'anima - passione, calcolo, brutalità, sentimentalità - e non già per indicare, come segno algebrico, il tale o tal altro principio psicologico che passa pel capo dell'autore, ma perché proprio continuano nel libro la Natura, perché proprio portano in sé creata con tutti i mezzi dell'arte letteraria la particolar vita sensuale, sentimentale, intellettuale, di esseri umani collocati nel centro della vita universa» (Capuana, 1898: 45). Ma se l'arte, annota l'autore, «crea individui non tipi» e se ogni individuo è «un mondo a parte, immensamente vario, quasi altrettanto infinito quanto l'universo», «l'arduo, il difficile sta nel penetrare nello scrutare quell'abisso e illuminarlo con la viva luce dell'arte» (Capuana, 1898: 46) La meta cui tendere è dunque «dipingere dal vero» $l^{\prime}$ «interno stato», mentre artista sarà colui che, come Verga, non soltanto ha «saputo compenetrarsi col personaggio da sparire dentro di lui», secondo il canone del metodo impersonale, ma soprattutto «ha saputo mettergli in bocca la parola giusta, rapida, incisiva, che condensa in poche sillabe lo infinito dell' anima» (Capuana, 1898: 183). È questo l'esito più maturo cui perviene la riflessione capuaniana: la scoperta di un individuo inconoscibile, il difficile tentativo, se non l'impossibilità, di esplorare l'«abisso» che abita l'animo umano sono categorie che già preludono al relativismo gnoseologico di tanta letteratura novecentesca.

Quella difficoltà di attraversare i territori impervi della perturbante prospettiva dell'ignoto, in cui è il segno del passaggio dall'osservazione del dato fisiologico allo scandaglio esistenziale, se è vera per la narrativa, cui tuttavia quell'indagine meglio si attaglia in funzione della vocazione analitica tipica del romanzo, lo è ancor di più per la tendenza alla sintesi del teatro che, nel restituire le complesse elucubrazioni del personaggio, impone uno scavo nella coscienza non soltanto da parte dello scrittore ma pure dell'attore cui è delegato il compito di tradurre in azione imperscrutabili moti dell'anima. Nella lucida consapevolezza di quel delicato processo di trasposizione, il 3 settembre 1892, da Roma, così Capuana scrive in una lettera inedita al fratello Francesco, impegnato a seguire le prove di una messa in scena catanese:

Caro Francesco 
Eccoti la copia di Malia coi tagli e le correzioni fattevi dopo le prime recite qui. [...] La prima donna deve stare attenta alle tre gradazioni del carattere di Iana. Ne $1^{\circ}$ atto è triste, pensierosa, ma fa sforzi per contenersi. Nel secondo è ammalata sotto l'influenza della crisi isterica e della creduta malìa. Nel terzo è guarita, ma è tormentata dai rimorsi. Sono tre aspetti diversi. L'insuccesso della prima sera qui tornentata dai rimsi. Sono tre ast al personaggio in tutti i tre atti.

Dopo lunghe trattative con i capocomici Francesco Pasta e Cesare Rossi, che rifiutano l'opera «perché assomiglia un poco alla Cavalleria rusticana» (Raya, 1984: 340), tanto da indurre l'autore ad affermare «c'è una una malìa contro Malia» (Raya, 1984: 343), Capuana affida il dramma alla compagnia di Giuseppe Pietriboni, certamente incapace di sostenere la difficoltà del testo, che lo mette in scena il 9 maggio 1892 al Teatro Nazionale di Roma con scarso successo, come si apprende dalle cronache del tempo. Fra queste ampia e dettagliata è quella dell'amico Giuseppe Alfredo Cesareo che, nell' articolo apparso il 15 maggio sulla «Tavola Rotonda», non manca di sottolineare gli errori imputabili all'interpretazione («tutti [...] fecero la loro parte, per parlare sotto metafora, caninamente»). Significativa l'annotazione relativa al personaggio di Jana, definita «creatura viva e stupenda»: «che bellezza di carattere quella Jana! Innocente appassionata, sincera, sempre uguale dal principio alla fine». Anche il cronista del «Folchetto», sia pure con accenti diversi, ravvisa la monotonia interpretativa della protagonista, sottolineando come il pubblico abbia ritenuto «di soverchio opprimente la figura della Jana, che piange e sospira sempre». Si comprende così l'opportunità dell'osservazione di Capuana nel raccomandare al fratello un'attenzione particolare per le diverse sfumature del carattere di Jana che la prima attrice evidentemente non seppe cogliere, restituendo al contrario una intonazione monocorde priva delle diverse tonalità attraverso cui si esplicita il segreto tormento della protagonista. «Questione d'interpretazione», si potrebbe affermare col Verga che, a proposito del dramma «intimo» In portineria, chiosava: «interpretazione da parte dello scrittore, dell'attore ed anche, vedi, del pubblico, che [...] dovrebbe portare una certa dose di osservazione, d'amore, e direi di collaborazione» (Raya, 1984: 242). Ulteriore passo verso l'attuazione dell'ideale della «verità» e della «semplicità» di contro alla «convenzione» e alla «retorica», una recitazione priva di accenti declamatori costituiva il traguardo da raggiungere nel processo di ammodernamento delle scene cui miravano i maestri del verismo e contemporaneamente anche la migliore sperimentazione teatrale europea, quella che a partire da Zola passa per Antoine, Brahm, i Meininger, fino a Stanislavskij. Un processo di deteatralizzazione già auspicato da Capuana nell'assiduità dell'esercizio critico del periodo fiorentino e messo in pratica in occasione della prova scenica di Giacinta allorché ribadisce la volontà di «semplificare l'azione», «sbarazzarla di quelle convenzioni che il lungo uso o l'abuso, anche di scrittori di grandissimo ingegno, hanno talmente mescolate con quelle che costituiscono l'essenza dell'arte drammatica da farle credere erroneamente della medesima natura di essa e perciò in egual grado rispettabili, anzi intangibili» (Capuana, 1999: 41). E che impone pertanto la riforma dei moduli recitativi della scuola declamatoria, la necessità di disfarsi di un arsenale di artifici e di effetti a favore di quei toni da colloquialità quotidiana che consentano all'attore di vivere la parte non di rappresentarla. "Questa commediola va recitata male per essere resa bene, cioè senza enfasi né effetti teatrali. Io voglio la stessa semplicità e la stessa naturalezza della gente che parli e si mova come i contadinie non sappia di recitare», aveva osservato ancor prima Verga a proposito della messa in scena di Cavalleria rusticana. E Capuana, qualche anno più tardi, nella prefazione alla commedia Serena:

ho voluto scrivere una commedia di soggetto moderno con antica semplicità, evitando di adoperare e i grandi e i piccoli artifici teatrali, diventati ormai ferri inservibili per l'uso e l'abuso che n'è stato fatto da secoli. [...] Per ciò essa non ha prime parti per comodo di una attrice o di un attore; per ciò, nel momento opportuno, quel che sembra il più dimesso e il più scolorito dei suoi personaggi assume, tutt'a un tratto, rilievo e importanza. Concepita con questi intendimenti, io preferisco di presentarla al pubblico su le pagine di una rivista prima che su le tavole del palcoscenico; se pure sarà possibile in Italia, nelle attuali condizioni dell'arte rappresentativa, trovare chi voglia coadiuvare un autore in un esperimento d'arte dove la semplicità dei mezzi sembra escludere qualunque lusinga alla abilità delle attrici e degli attori: quasi certi caratteri e certi sentimenti, che richiedono uno sforzo di delicata e pri, quasi interpretion interpretazione, non possano far rifulgere - e forse meglio di altri caratteri (Oliva, 1999: 165-166).

Uno «sforzo di delicata e profonda interpretazione» richiedeva pure il ruolo di Jana, personaggio che reca, celato dietro il cliché della donna vittima di una passione travolgente, quel germe della modernità destinato a contaminare consolidate convenzioni poetiche, sceniche e sociali. Un ruolo che soltanto la 'verità' di toni, mimica e costumi di Giovanni Grasso e Marinella Bragaglia, primi attori della «Compagnia drammatica dialettale siciliana» diretta da Nino Martoglio, poteva assolvere. È per questo che il 7 dicembre 1902 Capuana presenta all'ex puparo quel dramma che nella traduzione in dialetto siciliano e per i meriti di interpreti che si segnalavano «per forte semplicità e per intuito artistico» avrebbe calcato i palcoscenici più prestigiosi di tutto il mondo, inserendosi nella stagione aurea del teatro siciliano che la benemerita attività critica e di ricerca di Sarah Zappulla Muscarà e di Enzo Zappulla ha riportato alla luce nella sua autentica dimensione di esperienza fondativa della drammaturgia contemporanea. E si compie così l'ennesima svolta artistica capuaniana, quella che porta lo scrittore all'accettazione non pregiudiziale di quel teatro regionale inizialmente considerato, alla luce dell'esigenza dell'unificazione linguistica oltre che politica de nuovo Stato, una forma d'arte «inferiore per i mezzi che usa, inferiore pel suo contenuto che non esce e non può affatto uscire da una certa classe sociale, inferiore per le sue
Revista Internacional de Culturas y Literaturas abril 2011

ISSN: 1885-3625 
intenzioni artistiche che son messe al secondo, al terzo, all'ultimo posto nella mente dello scrittore» (Capuana, 1872: XXX), nel quale scorge ora l'unico mezzo per arrivare «a quel teatro nazionale, che non ha altra via di salvezza, se vuol essere opera d'arte $\mathrm{e}$ non opera d'artifizio» (Capuana, 1911: 74).

Sintesi della polarizzazione contenutistico-formale del filone 'appassionato' 'paesano' ma pure del passaggio dalla narrativa al teatro (per «la stessa formula d'arte» l'opera è inserita nella silloge di novelle Le Paesane), testo spartiacque fra vecchio e nuovo, Malìa accoglie le più innovative sollecitazioni culturali assorbite da Capuana alla fine del secolo, fra il crepuscolo di un'epoca e un'alba foriera di mutamenti, sconfinando nei territori imprevisti dell'esacerbato dolore esistenziale. Ma a un simile inatteso esito, che avrebbe sottoposto a verifica uno status quo cui appartiene lo stesso scrittore, forse Capuana non era preparato. Meglio dunque rifugiarsi nel consueto tragico epilogo, lasciando soltanto indovinare dalle crepe dell'edificio verista la cris irreversibile che attende l'individuo e la società borghese alle soglie della modernità.

\section{RIFERIMENTI BIBLIOGRAFIC}

AA. VV., Studi sulla cultura italiana tra Ottocento e Novecento, a cura di W. Tega, Bologna, Clueb, 1982.

AA. VV., Capuana Verista, Catania, Fondazione Verga, 1984.

AA. VV., Angelo Musco e il teatro del suo tempo, a cura di E. Zappulla, Giuseppe Maimone Editore, 1987.

AA. VV., Naturalismo e Verismo, Catania, Fondazione Verga, 1988

AA. VV., Capuana drammaturgo, critico, narratore, Catania, Teatro Stabile, 1991.

AA. VV., Il teatro verista. Atti del Congresso di Catania, 24-26 novembre 2004, Biblioteca della Fondazione Verga, vol. I, Catania, 2007.

Angelini, F., Teatro e spettacolo del primo Novecento, Roma-Bari, Laterza, 1988.

Antonucci, G., Storia della critica teatrale, Roma, Studium, 1990

Artioli, U., Teorie della scena dal naturalismo al surrealismo, Firenze, Sansoni, 1972.

Barbina, A., Teatro verista siciliano, Bologna, Cappelli Editore, 1970.

----, Capuana inedito, Bergamo, Minerva Italica, 1974.

Barsotti, A., "La Lupa" e "Malìa" in Verga drammaturgo. Tra commedia borghese e teatro verista siciliano, Firenze, La Nuova Italia, 1974.

Capuana, L., Il teatro italiano contemporaneo, Palermo, Pedone Lauriel, 1872.

----, Profili di donne, Milano, Brigola, 1877.

---, Giacinta, Milano, Brigola, 1879 (II ed. Catania, Giannotta, 1886; III ed. con Prefazione

a Neera ivi, 1889; IV ed. Milano, Cervieri, 1914).
----, Studi sulla letteratura contemporanea. Prima serie, Milano, Brigola, 1880.

----, Studii sulla letteratura contemporanea. Seconda serie, Catania, Giannotta, 1882 (rist. a cura di P. Azzolini, Napoli, Liguori, 1988).

----, Homo!, Milano, Brigola, 1883 (II ed., Milano, Treves, 1888).

----, Per l'arte, Catania, Giannotta, 1885 (rist. a cura di R. Scrivano, Napoli, Esi, 1994).

----, Ribrezzo, Catania, Giannotta, 1885.

----, Il piccolo archivio, Catania, Galatola, 1886.

----, Giacinta, commedia in cinque atti in prosa, Catania, Giannotta, 1890.

----, Malia, Roma, Tipografia Sinimberghi, 1891

----, Libri e teatro, Catania, Giannotta, 1892.

----, Profumo, Palermo, Pedone-Lauriel, 1892 (già a puntate in «Nuova Antologia», 1 luglio-1 dicembre 1890).

----, Le appassionate, Catania, Giannotta, 1893.

----, Malia, melodramma in tre atti, musica di F. P. Frontini, Bologna, Tedeschi, 1893.

----, Le paesane, Catania, Giannotta, 1894 (rist. a cura di E. Villa, Milano, Marzorati, 1974).

----, Gli “ismi" contemporanei, Catania, Giannotta, 1898 (rist. a cura di G. Luti, Milano, Fabbri, 1973).

----, Cronache letterarie, Catania, Giannotta, 1899.

----, Il marchese di Roccaverdina, Milano, Treves, 1901 (già a puntate in «L'Ora», 12 settembre-11novembre 1900, ma incompiuto).

----, Teatro dialettale siciliano, vol. I, Palermo, Reber, 1911 (contiene Malìa, Lu cavaleri Pidagna); vol. II, ibidem (contiene Bona genti, Ppi lu currivu); vol. III, ivi, 1912 (contiene Cumparaticu, 'Ntirrugatoriu, Riricchia); vol. IV, Catania, Giannotta, 1920 (contiene Lu Paraninfu, Don Ramunnu); vol. V, ivi, 1921 (contiene Quacquarà, Prima di li milli).

----, Serena, a cura di P. Marletta, Catania, Società di Storia patria per la Sicilia Orientale 1973.

----, Racconti, a cura di E. Ghidetti, Roma, Salerno editrice, 1973-1974.

----, Teatro dialettale siciliano, a cura di P. Mazzamuto, Catania, Giannotta, 1974.

----, Il Mulo di Rosa, a cura di V. Frosini, Catania, Società di Storia patria per la Sicilia Orientale, 1976.

----, Il Mulo di Rosa, a cura di V. Frosini, Catania, Società di Storia patria per la Sicilia Orientale, 1976 
----, Giacinta [1879], a cura di M. Paglieri, introduzione di G. Davico Bonino, Milano, Mondadori, 1980

----, Giacinta [1889], a cura di S. Zappulla Muscarà, Milano, Mursia, 1980.

----, Giacinta [1914], a cura di E. Ghidetti, Roma, Editori Riuniti, 1980.

----, Malìa, Catania, Teatro Stabile, 1990.

----, Malia, a cura di D. Morea, Napoli, Bellini, 1995.

----, Il Paraninfo, a cura di S. Zappulla Muscarà, Catania, C.U.E.C.M., 1996.

----, Malìa, a cura di S. Zappulla Muscarà, Mineo, Centro Nazionale di Studi Luig Capuana, 1997.

----, Teatro italiano, a cura di G. Oliva, Palermo, Sellerio, 1999.

----, Cronache drammatiche, a cura di G. Oliva, voll. 2, Edizione Nazionale dell'Opera omnia di Luigi Capuana, Roma, Salerno editrice, 2010.

----, Lettere al fratello Francesco, a cura di L. Marullo, prefazione di S. Zappulla Muscarà, in corso di stampa.

Colicchi, C., "De Sanctis, Capuana e la poetica del Verismo", in AA. VV., De Sanctis e il realismo, vol. II, Napoli, Giannini, 1978.

De Sanctis, F., L'arte, la scienza e la vita, a cura di M.T. Lanza, Torino, Einaudi, 1972

Di Blasi, C., Luigi Capuana, vita - amicizie - relazioni letterarie, Mineo, Edizione "Biblioteca Capuana", 1954.

Ghidetti, E., L'ipotesi del realismo. (Capuana, Verga, Valera e altri), Padova, Liviana Editrice, 1981 (II ed. Milano, Sansoni, 2000).

Giudici, P.E., "Luigi Capuana autore e critico drammatico", in Note e saggi di varia letteratura, Alcamo, Accademia di studi «Cielo d'Alcamo», 1953.

La Monaca, D., "La "narrazione" in scena: il teatro di Luigi Capuana", in Il marchese e la maestrina, Caltanissetta-Roma, Sciascia, 2003.

----, “L'«infinito dell'anima»: 'passione' e 'metodo' nei drammi in lingua di Luigi Capuana", in Sanfilippo, M.V., La duplice bestia nera di Luigi Capuana, Annali della Fondazione Verga, vol. I, Catania, 2008

Livio, G., La scena italiana. Materiali per una storia dello spettacolo dell'Otto e Novecento, Milano, Mursia, 1989.

----, "Il teatro del grande attore e del mattatore", in Storia del teatro modernoecontemporaneo. Il grande teatro borghese. Settecento-Ottocento, vol. II, Torino, Einaudi, 2000.

Madrignani, C.A., Capuana e il naturalismo, Bari, Laterza, 1970.

---, Angelini, F., Cultura narrativa e teatro nell'età del positivismo, Bari, Laterza, Teatro verista, a cura di V. Bracco, prefazione di U. Bosco, Brescia, La Scuola, 1975.
Malara, F., "Una professione inedita: il critico teatrale", in Storia del teatro moderno e contemporaneo. Il grande teatro borghese. Settecento-Ottocento, vol. II, Torino, Einaudi, 2000

Marchand, J.J., Édouard Rod et les écrivains italiens, Genève, Droz, 1980.

Martoglio , N., Tutto il teatro, a cura di S. Zappulla Muscarà, Roma, Newton \& Compton, 1996

Monti, S., Il teatro realista della nuova Italia (1861-1876), Roma, Bulzoni, 1978 Musumarra, C., "Capuana e la critica teatrale", in Critica letteraria, a. XXV, n. 94 1997.

Muscariello, M., "Una commedia tra le novelle: Malìa e Le Paesane di Luigi Capuana”, in AA. VV., Il teatro verista. Atti del Congresso di Catania, 24-26 novembre 2004, Biblioteca della Fondazione Verga, vol. I, Catania, 2007.

Nicastro, G., Teatro e società in Sicilia (1860-1918), Roma, Bulzoni, 1978

----, Scene di vita e vita di scene, Messina, Sicania, 1988.

Oliva, G., Capuana in archivio, Caltanissetta-Roma, Sciascia editore, 1979.

Palermo, A., "La formazione critica di Luigi Capuana", in Filologia e letteratura, a. X, fasc. IV, 1964 (poi in Lo spessore dell'opaco, Palermo, Flaccovio, 1979).

----, "Per una rivalutazione dell'ultimo Capuana", in AA. VV., L'illusione della realtà. Studi su Luigi Capuana, a cura di M. Picone e E. Rossetti, Roma, Salerno editrice, 1990.

----, "Il lungo interim di Capuana", in Ottocento italiano. L'idea civile di letteratura. Cattaneo, Tenca, De Sanctis, Carducci, Imbriani, Capuana, Napoli, Liguori, 2000.

Pellini, P., Naturalismo e verismo, Firenze, La Nuova Italia, 1998

Pullini, G., "Luigi Capuana: il teatro in lingua”, in Lettere italiane, n. 1, gennaio-marzo 1993.

Ragonese, G., “Capuana tra Positivismo e Naturalismo: «Il teatro italiano contemporaneo»", in AA. VV., Letteratura e critica. Studi in onore di Natalino Sapegno, vol. II, Roma, Bulzoni, 1975.

----, "Capuana tra Verga e d'Annunzio", in AA. VV., La presenza della Sicilia nella cultura degli ultimi cento anni, Palermo, Palumbo, 1977.

Raya, G., Carteggio Verga-Capuana, Roma, Edizioni dell'Ateneo, 1984.

Spinazzola, V., Verismo e positivismo, Milano, Garzanti, 1978.

Teatro dell'Italia unita, a cura di S. Ferroni, Milano, Il Saggiatore, 1980.

Tessari, R., "L'osservatorio teatrale di Capuana”, in AA. VV., Gesto e parola. Aspetti del teatro europeo tra Ottocento e Novecento, Padova, Esedra editrice, 1996. 
Tinterri, A., Il teatro italiano dal Naturalismo a Pirandello, Bologna, Il Mulino, 1990. Verga, G., La Lupa. Novella, dramma, tragedia lirica, a cura di S. Zappulla Muscarà, Palermo, Novecento, 1991.

Zappulla Muscarà, S., Zappulla, E., Sicilia: Dialetto e Teatro. Materiali per una storia del teatro dialettale, Agrigento, Edizioni del Centro Nazionale di Studi Pirandelliani, 1982.

----, Letteratura, Teatro e Cinema, Catania, Tringale, 1984.

----, Capuana e De Roberto, Caltanissetta-Roma, Sciascia, 1984.

----, Nino Martoglio capocomico, «Otto/Novecento», Varese, n. 1, a. VIII, gennaiofebbraio 1984 (poi Catania, C.U.E.C.M., 1985).

----, Nino Martoglio, Caltanissetta-Roma, Sciascia, 1985. Gramsci, A., Letteratura e vita nazionale, introduzione di E. Sanguineti, Roma, Editori Riuniti, 1987.

----, Zappulla, E., Musco. Immagini di un attore, Catania, Giuseppe Maimone Editore, 1987.

-----, Musco. Il gesto, la mimica, l'arte, Palermo, Novecento, 1987.

----, Zappulla, E., Giovanni Grasso il più grande attore tragico del mondo, Catania, la Cantinella, 1995.

----, Luigi Capuana e le carte messaggiere, Catania, C.U.E.C.M., 1996.

----, Le donne del teatro siciliano. Da Mimì Aguglia a Ida Carrara, Catania, la Cantinella, 2005.

----, "Il teatro siciliano in Spagna", in AA. VV., Italia-España-Europa Literaturas Comparadas, Tradiciones y Traducciones, XI Congreso Internacional de la Sociedad Española de Italianistas, coordinadores M. Arriaga Flóres, J.M. Estevéz Saá, D. Ramírez Almazán, L. Trapassi, C. Vera Saura, Sevilla, Arcibel Editores, 2005.

----, "La vocazione universale di Mimì Aguglia", in AA. VV., Mujeres E Máscaras. Ficción, simulación \& espectáculo, Editor@s V. González Martín, M. Arriaga Flóres, C. Aramburu Sánchez, M. Martín Clavijo, Sevilla, Arcibel Editores, 2010.

Zola, É., Le naturalisme au théâtre, Paris, Charpentier, 1881 (traduzione italiana Il naturalismo a teatro, a cura di G. Liotta, Bologna, Edizioni Libricooper, 1983). 